\title{
On the Representation of Human Motions and Distance-based Retargeting
}

\author{
Simon B. Hengeveld* A. Mucherino, ${ }^{*}$ \\ *IRISA, University of Rennes 1, Rennes, France. \\ simon.hengeveldeirisa.fr, antonio.mucherinodirisa.fr
}

\begin{abstract}
Distance-based motion adaptation leads to the formulation of a dynamical Distance Geometry Problem (dynDGP) where the involved distances simultaneously represent the morphology of the animated character, as well as a possible motion. The explicit use of inter-joint distances allows us to easily verify the presence of joint contacts, which one generally wishes to preserve when adapting a given motion to characters having a different morphology. In this work, we focus our attention on suitable representations of human-like animated characters, and study the advantages (and disadvantages) in using some of them. In the initial works on distance-based motion adaptation, a $3 n$ dimensional vector was employed for representing the positions of the $n$ joints of the character at a given frame. Here, we investigate the use of another, very popular in computer graphics, representation that basically replaces every joint position in the three-dimensional space with a set of three sorted Euler angles. We show that the latter can in fact be useful for avoiding some of the artifacts that were observed in previous computational experiments, but we argue that this Euler-angle representation, from a motion adaptation point of view, does not seem to be the optimal one. By paying particular attention to the degrees of freedom of the studied representations, it turns out that a novel character representation, inspired by representations used in structural biology for molecules, may allow us to reduce the character degrees of freedom to their minimal value. As a result, statistical analysis on human motion databases, where the motions are given with this new representation, can potentially provide important insights on human motions. This study is an initial step towards the identification of a full set of constraints capable of ensuring that unnatural postures for humans cannot be created while tackling motion adaptation problems.
\end{abstract}

\section{INTRODUCTION}

$\mathbf{M}$ OTION adaptation is a fundamental problem arising in computer graphics [6], [7], [8]. From a given motion for a given character, the interest lies in finding the way to impose the same (or a very "similar") motion to another character having a different morphology. The implications that a robust solution to this problem can give in the context of computer graphics are evident and include, for example, the partial automation in the production of animated pictures, as well as the conception and development of computer games. In the context of computer graphics, this problem is also known as motion retargeting.

We consider a simple undirected graph $G=(V, E)$ to represent the skeletal anatomy of our characters to be animated. The vertex set $V$ of the graph contains the joints of the character, while edges in $E$ represent rigid "bars" between some pairs of joints, that in this context are often referred to as character bones. In this work, we will focus our attention on graphs $G$ that are trees, where every joint has one unique parent joint. In fact, a tree $G$ is particularly suitable to represent the skeletal structure of human bodies. Together with $G$, the representation of our characters is generally integrated with the function

$$
\chi: v \in V \longrightarrow \chi(v) \in \mathbb{R}^{3},
$$

which assigns a three-dimensional position, w.r.t. its own parent, to every joint of the character. We remark that the function $\chi$ not only encodes the initial posture of the character (i.e. the relative position of all character joints in absence of movement), but it also implicitly provides information about the morphology of the character. In fact, for every bone $\{u, v\} \in E$, where the joint $u$ is parent of the joint $v$ in the tree $G$, the real value $\|\chi(v)\|$ corresponds to the length of the bone $\{u, v\}$ (the symbol $\|\cdot\|$ represents the Euclidean norm). Notice that, in the context of graph rigidity [1], [9], the pair $(G, \chi)$ is also called a skeletal structure. An example of skeletal structure for a human character is given in Fig. 1.

Motion adaptation asks, given a motion and a character $(G, \chi)$, whether it is possible to adapt this motion so that a different character $(G, \hat{\chi})$, having the same skeletal anatomy $G$ but a different morphology $\hat{\chi}$, can actually perform the same motion [6]. This is not a trivial problem, because even small changes in the morphology can make the original movement "look" different to the viewer. In particular, joint contacts play a very important role: joint contacts that are not preserved from the original motion are likely to give the viewer the impression that the motion is "different"; the same impression can be given by a motion performed by a morphological different character where new joint contacts, originally not appearing in the motion, are introduced. We point out that the concept of contact is closely related to the concept of proximity (or distance).

This work elaborates on some previous research on distancebased motion adaptation [3], [17]. The main idea is to represent the character animations by an alternative representation where the distance information is exploited. Inter-joint distances, in fact, can at the same time encode the morphology of the character (given by the function $\chi$ ), as well as the relative movements of the joints that are not connected by an edge of $G$. This alternative representation seems to be very convenient to the purposes of motion adaptation, because it allows for an easy detection of joint contacts. This is done 
by the simple verification of the values of the corresponding distances (the contact detection is otherwise not trivial in other usually employed representations for the motions, the ones we will discuss in Section II).

Evolving inter-joint distances, however, do not allow for defining the most efficient representation of a character motion. Even when making the hypothesis that all involved distances are known at a high precision level, the set of evolving distances are likely to carry highly redundant information, and any modification on one single distance would imply the infeasibility of the distance set. Therefore, we use distance-based representations of the motions only for generating instances of the dynamical Distance Geometry Problem (dynDGP) [16] where the distance information is extracted, at the same time, from the original motion, as well

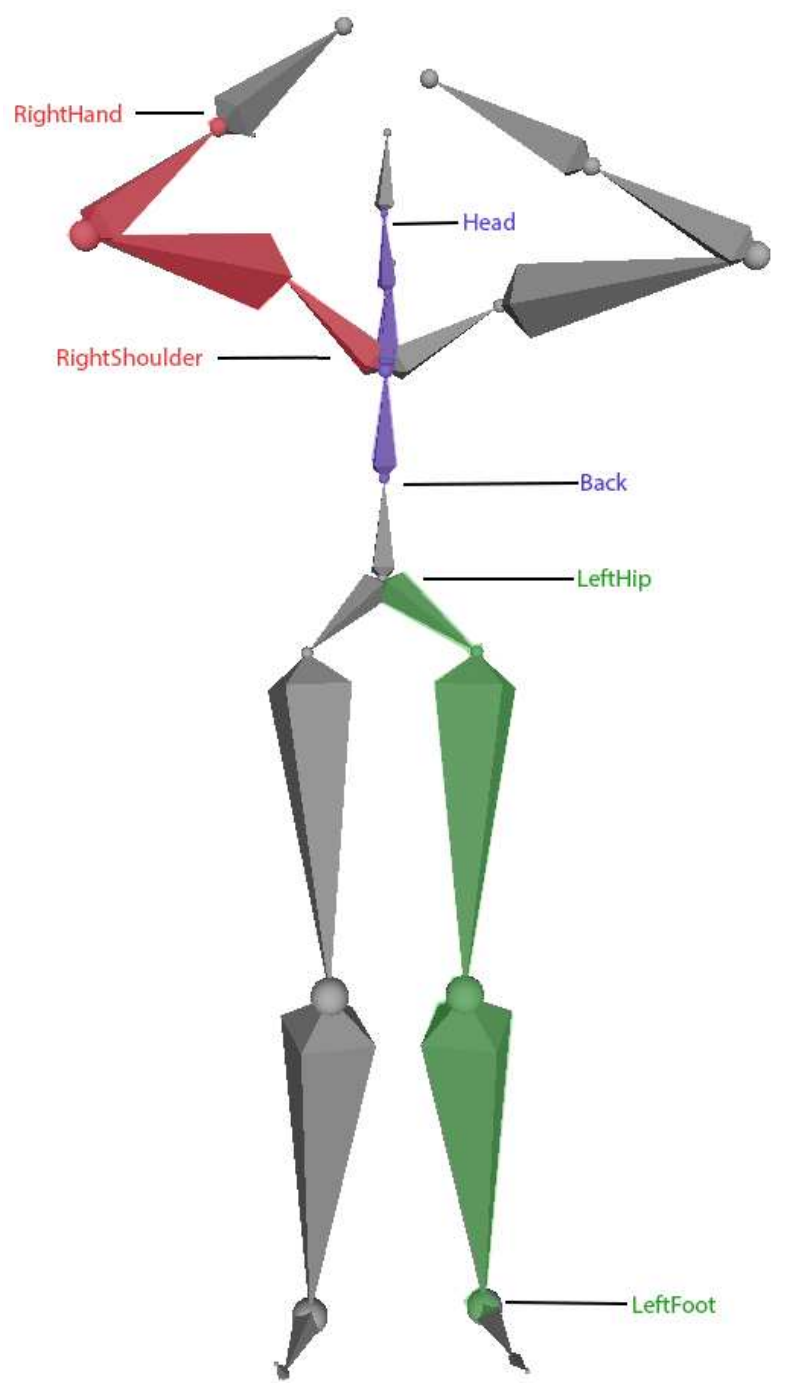

Fig. 1. An example of skeletal structure $(G, \chi)$. Marked in red, violet and green, the joints and bones involved in the computation of the vector and torsion angles for, respectively, the joint representing the RightHand, the Head and the LeftFoot of the human character. as from the target morphology $\hat{\chi}$. The solution to the dynDGP instance will consist in a motion for the character $(G, \hat{\chi})$ where the error on the given distances is minimized. Naturally, some distances (such as the bone lengths indicated by the function $\hat{\chi})$ have a higher importance w.r.t. others.

An important part of this work is devoted to the representations employed for the resulting motions. The solution to the generated dynDGP instance allows us to convert the high-redundancy and sensitive distance-based representation, which is however able to efficiently control character interjoint proximity, to another "more convenient" representation. In the initial works in [17], every joint was represented with its three-dimensional coordinates in the Euclidean space, but it was subsequently shown that the use of this representation can potentially introduce artifacts in the resulting motions [3].

In this work, we make two steps forward in the identification of an optimal representation for the character motions. First, in the experiments we will present on motion adaptation, we will use the typical (and very popular) representation used in the context of computer graphics [13], which is based on the rotations given by three Euler angles on every character bone. We will show that some of the previously observed artifacts disappear with the employment of this Euler-angle representation. Second, we will present a study on the degrees of freedom of different possible representations for the motions, which shows that the Euler-angle representation is not the optimal one in terms of degrees of freedom. In fact, this representation does not ensure that there is a bijective correspondence between character postures and variable values. Therefore, we propose another representation, inspired by a molecular representation that is widely used in the context of structural biology. By using this new representation, we performed a statistical analysis on a database of human character motions, with the final aim of deriving simple constraints capable to delimit the postures that are natural for a human character.

The rest of the paper is organized as follows. Section II will discuss different existing mathematical representations of character motions, while Section III will introduce our novel character representation inspired by the representation of biological molecules. By taking into consideration the very popular character representation which associates three Euler angles to each character joint, we will revisit in Section IV our distance-based motion adaptation approach, and some computational experiments will be presented in Section IV-A. By taking into consideration, instead, our representation of the motions introduced in Section III, we will present a statistical analysis on some of the variables used in this representation. Finally, Section VI will conclude this article with some directions for future works.

\section{A MATHEMATICAL DESCRIPTION OF MOTIONS}

In the wide computer graphics literature on motion retargeting, it is not common to find rigorous descriptions of the objects that come to play. An initial attempt can be found in [15]. In this section, we briefly re-propose the mathematical 
description given in the previous reference, and extend it to the purposes of our article.

Let $G=(V, E)$ be a simple undirected graph representing the skeletal anatomy of the characters. The pair $(G, \chi)$, coupling the graph $G$ with the function $\chi: v \in V \rightarrow \chi(v) \in \mathbb{R}^{3}$, represents a skeletal structure, which completes the description of the character with its morphology.

We point out that the graph $G$ generally contains a fictive root joint $v_{0} \in V$ that is always associated to the origin (i.e. $\left.\chi\left(v_{0}\right)=(0,0,0)\right)$ of the three-dimensional Euclidean space where the motions take place. The joint $v_{0}$ is naturally not part of character; it is only used to encode the global orientation of the character in its environment. With this constraint on the root joint $v_{0} \in V$, and by using the values $\chi(v)$ for all other joints $v \in V$, a special realization $x$ of $G$ can be immediately derived. Let $p: v \in V \backslash\left\{v_{0}\right\} \rightarrow p(v) \in V$ be the function that assigns the parent to each vertex of $G$ (exception made for the root $v_{0}$ ). The realization $x$ can therefore be constructed as follows:

$$
x: v \in V \longrightarrow \begin{cases}(0,0,0) & \text { if } v=v_{0}, \\ \chi(p(v))+\chi(v) & \text { otherwise. }\end{cases}
$$

This graph realization corresponds to the character posture in absence of any movement, and we will refer to this special posture as the posture 0 .

One trivial representation of the motions is the one consisting in extending the function $\chi$ (or similarly, the function $x$ ) to the several time frames forming the motion (instead of using them for the definition of posture 0 only). This representation uses three variables per joint, and therefore it gives $3|V|$ degrees of freedom to the character. We point out, however, that this representation has a main drawback: when the values of $\chi(v)$ can vary over time for every vertex $v \in V$, the length $\|\chi(v)\|$ of the bones $\{u, v\} \in E$ are subject to change over time. We remark that this is an useless degree of freedom, that is likely to spoil the representations every time it is not made sure that all length values $\|\chi(v)\|$ remain constant during the motions.

A popular way to represent the motions consists instead in assigning the three Euler angles $\theta$ (pitch), $\phi$ (roll) and $\eta$ (yaw) to every bone of the skeletal structure representing our character [5]. In this representation, the bone lengths $\|\chi(v)\|$ are constant by definition, while the three Euler angles are in charge to rotate the bones $\{u, v\} \in E$ in order to identify the position of the joint $v$ w.r.t. the joint $u$ (where $u$ is the parent of $v$ in $G$ ). In other words, the Euler-angle representation ensures that the character morphology $\chi$ remains constant during the motion.

Posture 0 corresponds in this representation to a list of Euler angles (the triplet of angles $\theta, \phi$ and $\eta$ for each bone in the skeletal structure) that are equal to 0 . More specifically, the character motion can be encoded as a sequence over a predetermined number $m$ of frames $t \in T$, with $T=\{1,2, \ldots, m\}$, of the Euler angles for every bone $\{u, v\} \in E$ forming the animated character:

$\rho:(v, t) \in V \backslash\left\{v_{0}\right\} \times T \rightarrow\left(\theta_{p(v), v}^{t}, \phi_{p(v), v}^{t}, \eta_{p(v), v}^{t}\right) \in[0,2 \pi)^{3}$.
We point out that the order for the three Euler angles is generally not fixed, and may be specific to the joint; for simplicity, we will suppose in the following that this order is constant: first $\theta_{p(v), v}^{t}$, then $\phi_{p(v), v}^{t}$, finally $\eta_{p(v), v}^{t}$. This order does not have any impact in our discussion. A more important remark is that, given $\rho$, the realization $x$ in Equ. (1) can be simply derived [4].

Notice that the total number of bones in the character is $|V|-1$, because $G$ is a tree: the number of degrees of freedom for the two representations $x$ and $\rho$ is essentially the same. This simple fact immediately implies that $\rho$ is not an optimal representation for the motions. Apart from some drawbacks already discussed in [4] about the singularity and the accuracy of this representation, we can remark that the set of Euler angles gives to the animated characters unnecessary degrees of freedom. This claim is supported by the following two observations. First of all, there always exist different triplets of Euler angles for a joint $v$ that, when applied to the bone $\{p(v), v\}$, can place this joint in the same position [22]. Secondly, not all triplets of possible Euler angles correspond to natural human postures (imagine for example the postures where there are obtuse angles at knees or elbows). In [12], a specific representation was proposed for the human shoulders where scapulo-thoracic constraints and joint sinus cones are used to cope with unrealistic postures at the upper part of the body that a basic Euler-angle representation may give. In spite of these observed limitations, the function $\rho$ has remained predominant in widely-used file formats for character motions [13].

\section{A NOVEL REPRESENTATION}

In this section, a new character representation for which we are able to count a smaller number of degrees of freedom is presented. To this aim, we introduce the two new angles $\zeta_{v}$ and $\omega_{v}$, that will sometimes replace the triplet of Euler angles employed in the function $\rho$ for the representation of a joint. Differently from a Euler angle, which depends only on the joint $v$ itself and on its parent $p(v)$, the vector angle $\zeta_{v}$ depends on $v, p(v)$, as well as on $(p \circ p)(v)$. Moreover, the torsion angle $\omega_{v}$ depends on $v, p(v),(p \circ p)(v)$, as well as on $(p \circ p \circ p)(v)$.

The reader may have noticed that, in spite of this extra dependence, only the main joint $v$ is indicated as a subscript of the angle names $\zeta_{v}$ and $\omega_{v}$; this is done in order to have a lighter notation. However, it will be supposed that, every time these two angles are taken into account, the necessary ancestors of $v(p(v),(p \circ p)(v)$, and finally $(p \circ p \circ p)(v)$ for $\omega_{v}$, all exist.

Definition 1 Given a skeletal structure $(G, \chi)$ and one realization $x$, the vector angle $\zeta_{v}$ for the joint $v$ in this realization is the smallest angle formed by the line passing through $x((p \circ p)(v))$ and $x(p(v))$, and the line passing through $x(p(v))$ and $x(v)$.

We can remark that variations on values of the vector angle $\zeta_{v}$ imply movements of the joint $v$. However, as for the Euler 


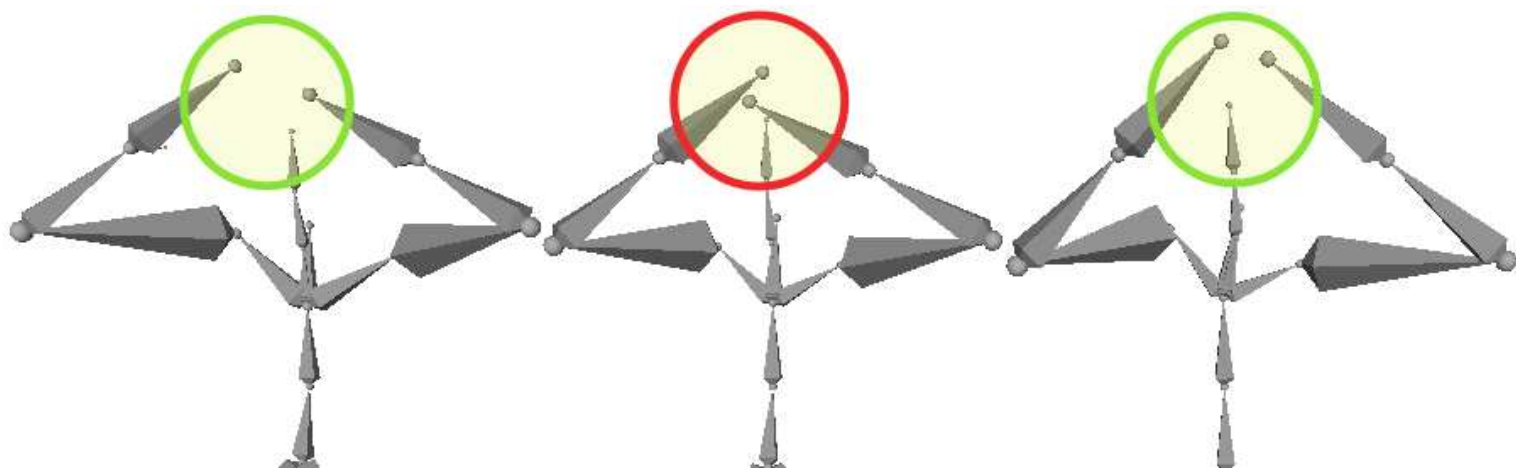

Fig. 2. A key frame for the macarena dance. From left to right: the posture in the original frame; the posture modified by simply transferring the original Euler angles to the different morphology; the posture obtained by our distance-based motion adaptation. The shoulders are $30 \%$ shorter in the target character. The distance between the two hands and their distance to the head are well preserved in our solution.

angles (because three of them are necessary to reconstruct the motion for each joint), one vector angle, alone, cannot be used to fully represent the motion of $v$. We couple therefore the $\zeta_{v}$ angle with a torsion angle.

Definition 2 Given a skeletal structure $(G, \chi)$ and one realization $x$, the torsion angle $\omega_{v}$ for the joint $v$ in this realization is the smallest angle formed by the plane defined by $x((p \circ p \circ p)(v)), x((p \circ p)(v))$ and $x(p(v))$, and the plane defined by $x((p \circ p)(v)), x(p(v))$ and $x(v)$.

In the hypothesis the realization $x$ preserves every bone length $\|\chi(v)\|$ (such as in the realization constructed in Equ. (1)), then, for every joint $v$ having at least 3 ancestors, we can identify its position in space by using the information about its distance $\|\chi(v)\|$ to the parent, the vector angle $\zeta_{v}$ and the torsion angle $\omega_{v}$ [11]. Again, three real-valued variables come to play per joint. However, in our case, the distance $\|\chi(v)\|$ is supposed to be constant in the skeletal structure $(G, \chi)$, reducing in this way the degrees of freedom per joint to 2 .

In the following, we will refer to our representation, which makes use of the two angles $\zeta_{v}$ and $\omega_{v}$, as the "vector-torsion angle representation". Ours is not a completely original representation. The two angles $\zeta_{v}$ and $\omega_{v}$ are commonly used in the context of structural biology to represent molecular conformations [2]. Exclusive vector-torsion angle representations are bijective, in the sense that any representation with a different set of angle values corresponds to a different conformation, and vice versa. When comparing with this different biological application, we can notice that the underlying graph $G$ has essentially the same features, even if its vertices represent atoms and not joints, while the morphology reflects the chemical bonds between pairs of atoms in the molecule. There are however two important differences in the two applications. First, in the "classical" backbone representation for special molecules named proteins, the vector angle $\zeta_{v}$ is also fixed (and not only the bond length $\|\chi(v)\|$ ), resulting in one unique degree of freedom per each quadruplet of consecutive atoms of the protein. In order to allow for wider movement possibilities, however, we cannot impose this constraint to our animated characters.

Secondly, and more importantly, molecular conformations do not need, in general, to be anchored to the environment. Several software tools for molecular visualization actually exploit this fact to allow the user to translate and rotate the molecular conformations as one pleases. This is not the case for our animated characters. As described in Section II, the fictive vertex $v_{0} \in V$ is used for encode the displacement, as well as the rotation, of the character w.r.t. the origin of its environment.

Therefore, we propose to combine this vector-torsion angle representation of the character with the Euler-angle representation, and to use one or another representation depending on the vertex $v \in V$. If $v$ is the only child of $v_{0}$, for example, there are two main reasons for choosing the triplet of Euler angles for its representation: first of all, $v$ does not have enough ancestors for the vector-torsion representation; secondly, the triplet of Euler angles can provide the global orientation of the joint in the character environment.

Let $R \subset V$ be the subset of joints that admit less than three ancestors. We propose the use of the following function for the representation of character motions:

$$
\begin{aligned}
& \rho^{\prime}:(v, t) \in V \backslash\left\{v_{0}\right\} \times T \longrightarrow \\
&\left\{\begin{aligned}
\left(\theta_{p(v), v}^{t}, \phi_{p(v), v}^{t},\right. & \left.\eta_{p(v), v}^{t}\right) \\
\left(\zeta_{v}^{t}, \omega_{v}^{t}\right) & \left.\in[0,2 \pi)^{3}, \quad \text { if } v \in R, 2 \pi\right)^{2}, \quad \text { if } v \in V \backslash R .
\end{aligned}\right.
\end{aligned}
$$

Fig. 1 depicts an example of skeletal structure for a human character (the joint $v_{0}$ does not appear in the figure). With the different colors, we have marked the joints and bones involved in the definition of the vector and torsion angles. For example, the position of the joint named LeftFoot (see the part marked in green in the figure) can be reconstructed by using the constant length of the bone joining the LeftFoot with its parent joint, plus the vector and torsion angles obtained by taking into consideration the other two immediate ancestors. The total number of degrees of freedom is $3(|R|-1)+2(|V|-|R|)$, which is smaller and at most equal to $3(|V|-1)$ when no leaves in $G$ admit at least 3 ancestors. 

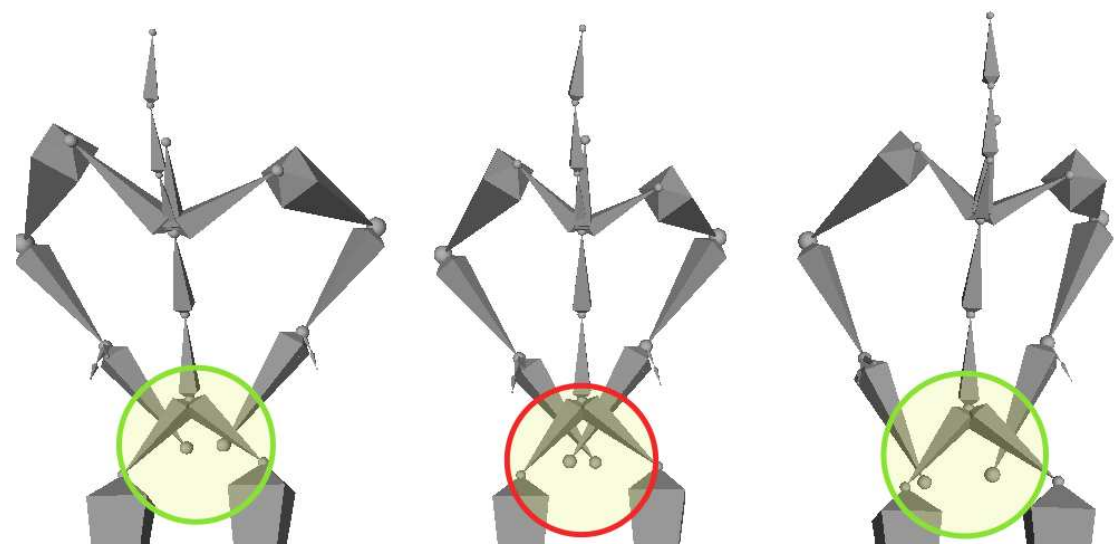

Fig. 3. Another key frame for the macarena dance. From left to right: the posture in the original frame; the posture modified by simply transferring the original Euler angles to the different morphology; the posture obtained by our distance-based motion adaptation. The shoulders are $30 \%$ shorter in the target character. The distance between the two hands does not exactly correspond to the original distance in our solution, but overall set of local distances around the character hands are well preserved, allowing the viewer to essentially perceive the same posture.

\section{THE DISTANCE-BASED APPROACH TO MOTION ADAPTATION REVISITED}

In motion adaptation [6], the interest is in adapting an original motion, given for the character $(G, \chi)$, to a character having a different morphology $(G, \hat{\chi})$ (notice that the anatomy $G$ is a constant). In this section, we will use the popular function $\rho$ to represent the character motions (see Section II). In order to adapt the motion to the character $(G, \hat{\chi})$, a new function $\hat{\rho}$ needs to be defined, where the set of Euler angles, related to each character joints, are adapted to take into consideration the modifications in the character morphology, while trying to preserve as much as possible the original motion.

This work develops on the distance-based approach for motion adaptation previously presented in [3], [17], where dynamical distance geometry plays a main role [19], [23]. We exploit the set of inter-joint distances extracted from the original motion $\rho$ of $(G, \chi)$ to control the proximity of the joints that are not connected by a bone in $G$. These distances allows us in fact to verify whether there are existing joints contacts in the original motion; and whether new (undesired) self-contacts can potentially be introduced while adapting the motion.

One of the main extensions w.r.t. the initial version of this distance-based approach consists in realizing the obtained motions directly in the space of Euler angles. In technical terms, instead of computing the coordinates, over time, for all the vertices of $G$ in $\mathbb{R}^{3}$ (see Equ. (1)), it is now possible to directly define the function $\rho$ to represent the adapted motions. Bond length fluctuations, observed in the experiments in [3], are in this way avoided by definition of the Euler-angle representation.

The first step in our distance-based approach consists in generating a dynDGP instance for which the solutions are the adapted motions [16], [17]. The distance information is extracted from the original motion $\rho$, as well as from the target morphology $\hat{\chi}$. From $\rho$, all inter-joint distances, at every frame $t \in T$, can be computed, including the original bone lengths $\|\chi(v)\|$. Even if the dynDGP is NP-hard in general [21], it is trivial to reconstruct, from this set of distances evolving over time, the original function $\rho$ (modulo equivalent Eulerangle representations) [10]. Naturally, our interest is rather in constructing a new function, corresponding to the animation adapted to the morphology of the target character.

One initial manipulation that one can consider to perform, in distance space, consists in replacing all bone lengths with the new values $\|\hat{\chi}(v)\|$. However, this simple modification is likely to introduce large errors in the other involved distances, the ones that actually describe the motion. Therefore, while changing the bone lengths, we also modify accordingly other distances that can be computed from the original motion. We follow the procedure detailed in [17]. We initially compute all shortest paths $P_{u v}=\left\{w_{1}, \ldots, w_{k}\right\}$ between pairs of distinct vertices, where $w_{1}=u, w_{k}=v$ and, for every $i=1, \ldots, k-1$, we have $\left\{w_{i}, w_{i+1}\right\} \in E$ (notice that the term "shortest" makes reference to the number of edges that need to the crossed by the path to walk from the vertex $u$ to the vertex $v$ of the graph). We refer to the sum of the edge weights (bone lengths) over a path $P_{u v}$ as the "weight" $\tau_{u v}$ of the shortest path $P_{u v}$, computed as:

$$
\tau_{u v}=\sum_{i=1}^{\left(\left|P_{u v}\right|-1\right)}\left\|\chi\left(w_{i}\right)-\chi\left(w_{i+1}\right)\right\| .
$$

The dynDGP instance is represented by a simple weighted undirected graph $H=\left(V \backslash\left\{v_{0}\right\} \times T, E_{H}, d\right)$, where $d$ is composed by the two real-valued functions, the $\delta$ function, and the $\pi$ function. The former function associates a distance to the subset of vertex pairs in $H$, as follows:

$$
\begin{aligned}
\delta:\{ & \{u, q\},\{v, t\}\} \in E_{H} \longrightarrow \\
& \begin{cases}\frac{\hat{\tau}_{u v}}{\tau_{u v}}\left\|x_{u}^{t}-x_{v}^{t}\right\|, & \text { if } t=q, \\
\left\|x_{u}^{q}-x_{v}^{t}\right\|, & \text { if } u=v \text { and } q=t-1,\end{cases}
\end{aligned}
$$



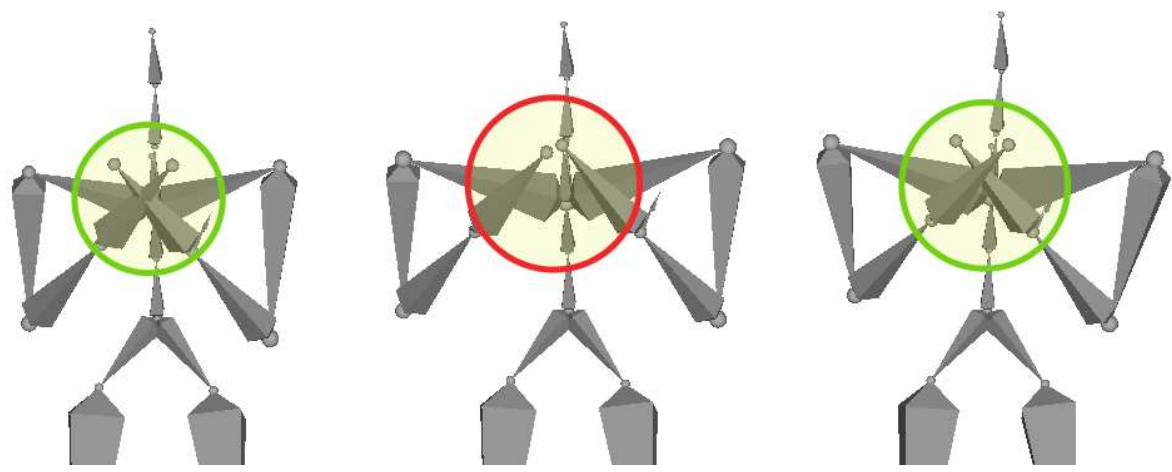

Fig. 4. A key frame for the character feeling cold and hence rubbing its hands. From left to right: the posture in the original frame; the posture modified by simply transferring the original Euler angles to the different morphology; the posture obtained by our distance-based motion adaptation. The shoulders are $30 \%$ longer in the target character. In the angle-transfer solution, the two hands can hardly touch one another.

where $x_{v}^{t}$ represents the position of the joint $v$ at time $t$ indicated by the original function $\rho$. For all situations that are not considered in the definition of $\rho$ (such as for example $q=t-2$ ), we suppose that the corresponding edge is not included in $E_{H}$. We point out that the inter-frame distances were originally not taken into consideration in the distancebased approach: this is another novelty introduced in our revisited version.

The $\pi$ function indicates the importance (the priority) of the distances given by the $\delta$ function. The first criterion to define the distance priorities is based on the fact that distances between joints that are closer in the skeletal structure (i.e. corresponding to shortest paths $P_{u v}$ over fewer bones) can be approximated better than others. For example, bone lengths can be exactly transformed into the ones of the target morphology $\hat{\chi}$, while the distances between two leaves of $G$ (such as a hand and a foot) rather represent rough approximations of the actual distances.

Our revisited approach improves this priority calculation by exploiting the information given by the interaction distance [15] between two joints $u$ and $v$ at a certain time frame $t \in T$. The interaction distance allows us to predict the distance that the two joints will have if their current relative movement (computed by comparing the current joint positions with the positions of the same joints at the previous frame) will not change in the subsequent frames. When two joints are moving one towards the other, their relative distances over time are in fact important for performing the adaptation, because they can guide the movement towards a joint contact that we want to preserve, or to avoid potential self-contacts in approaching joints. Therefore, we also assign a higher priority to the distances between joints $u$ and $v$ for which the corresponding interaction distance $I(u, v, t)$, at frame $t$, is smaller than a given positive threshold $\Delta$.

Finally, we give the maximal importance (priority 1) to the newly introduced inter-frame distances. To sum up, our $\pi$ function has the following form:

$$
\begin{aligned}
\pi: & \{\{u, q\},\{v, t\}\} \in E_{H} \longrightarrow \\
& \begin{cases}1, & \text { if } q=t-1, \\
1, & \text { if } q=t \text { and }(u=p(v) \text { or } I(u, v, t)<\Delta), \\
\left(P_{\max }-\left|P_{u v}\right|+2\right) / P_{\max }, & \text { otherwise, }\end{cases}
\end{aligned}
$$

where $P_{\max }$ is the maximal length (in terms of number of crossing edges) for a path on the graph $H$.

In this work, we find solutions to the dynDGP instance represented by the created graph $H$ by solving an optimization problem whose stress function measures the violation on the given distance constraints, where the violations on distances having higher priority give a higher contribution to the stress value (see [19] for a detailed description). We remark that, even if our current implementation is based on the Eulerangle representation $\rho$, it is necessary to compute the absolute positions $x_{v}^{t}$ of every joint at every frame in order to calculate the value of the stress function. Since our dynDGP instance can be easily separated in several sub-instances representing the postures over time of our animated characters, we make the choice initially proposed in [17] to optimize the stress function frame by frame, by considering only the local distance information, and by exploiting the result obtained during the optimization process at the previous frame.

We run a non-monotone spectral projected gradient method for performing the adaptation of every frame of the motions by using the available distance information. Since, in the motions, the character postures slightly change from one frame to the subsequent one, the spectral method takes as a starting point the posture obtained at frame $t-1$ to optimize the stress function for the current frame $t$. The only exception is given by the very first frame, where we select as a starting point the first frame of the original motion.

The next section presents some computational experiments performed with our revisited distance-based approach.

\section{A. Distance-based motion adaptation in practice}

We implemented our distance-based approach to human motion adaptation in the Java programming language. In this 

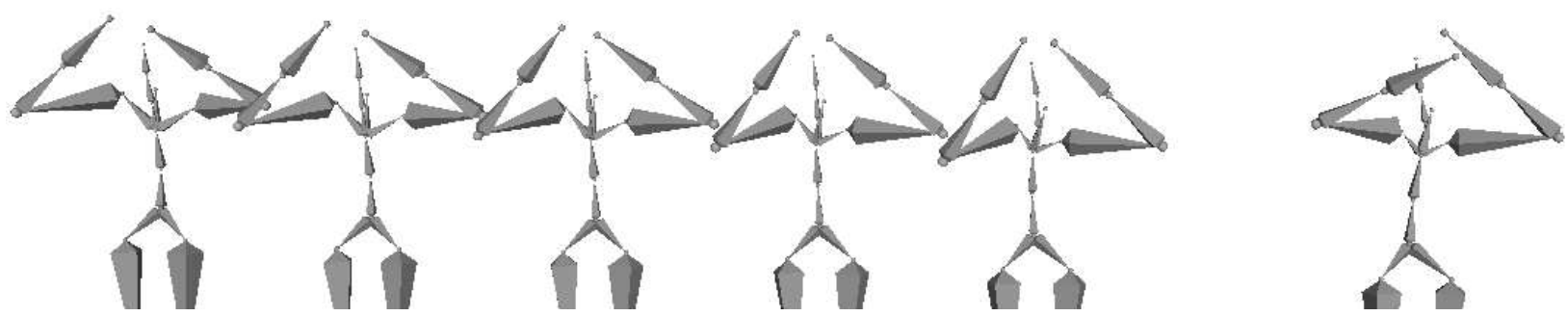

Fig. 5. On the right-most image, the result of retargeting the macarena dance key frame shown in Fig. 2 when the shoulders are $50 \%$ shorter. The sequence on the left-side of the figure shows the 5 steps to move from a $10 \%$ modification to the final $50 \%$ modification, with a change of $10 \%$ per step, necessary to obtain a good-quality solution when the changes in the morphology are more important.

section, we present some computational results where we have selected two motions from the Graphics Lab Motion Capture Database, ${ }^{1}$ provided by the Carnegie Mellon University. Our Java code accepts a BVH file in input, containing the original motion, and outputs the retargeted motion in the same format. The pictures presented in this article were created with the free software Blender ${ }^{2}$. We will not report the numerical values of the stress function for the frames of the obtained motions because they do not always reflect the viewer perception on the correctness of postures or movements.

The first motion that we consider is the famous "macarena" dance (database entry code 135_35, see Fig. 2). At a certain point, the character is supposed to place its both hands on its head. The original frame is shown in the left-most image in Fig. 2. We can notice that, when the shoulders are reduced in length $(-30 \%)$ and the original Euler angles are simply transferred to the new character (see central image), the two hands approach too much, and if one imagines where the head of the character is supposed to be, the viewer has the impression that the hands actually penetrate the character head. In the right-most image in Fig. 2, our solution shows a correct adaptation of the Euler angles to preserve the interjoint distance between the hands.

In the same motion, the hands of the character are placed on its back a few frames later. The same change in the morphology implies another undesired effect in the animation that is obtained by simply transferring the Euler angles. Fig. 3 compares the original, the angle-transfer result and the result obtained with our approach. Again, one may well confuse the original posture with our result if they were not simultaneously visible to the viewer.

The next motion shows a character that feels like it is cold: to warm up the hands, it rubs them together (entry code 79_68, see Fig. 4). When the new character with shorter shoulders tries to warm up the hands, its two hands are actually too far for performing a proper rubbing movement in the angletransfer posture (see central image in Fig. 4). The right-most image shows instead that this artifact is not present in our retargeted motion.

\footnotetext{
${ }^{1}$ https: //mocap.cs.cmu.edu

${ }^{2}$ https: / / www.blender.org
}

All adapted motions can be viewed on the YouTube video ${ }^{3}$ of a talk given, a few months before the publication of this ar-

${ }^{3}$ https : / / www youtube. com/watch?v=UWf 7 z 1 cDWmc

Head joint

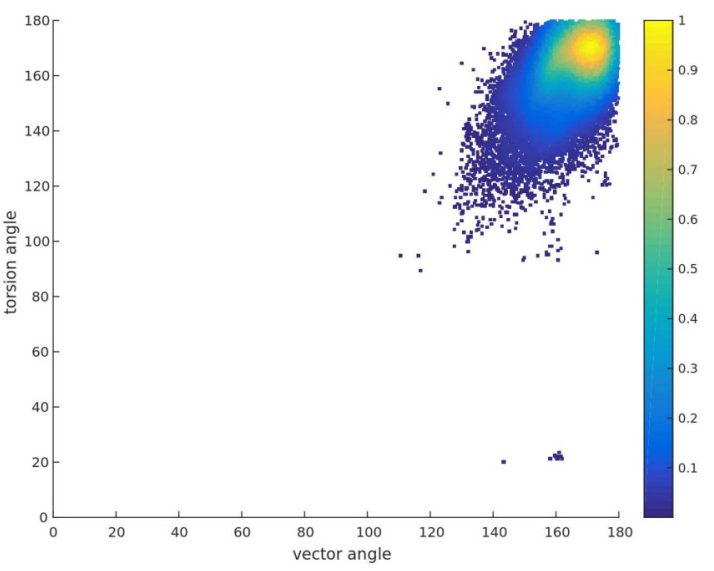

Hand joint (left or right)

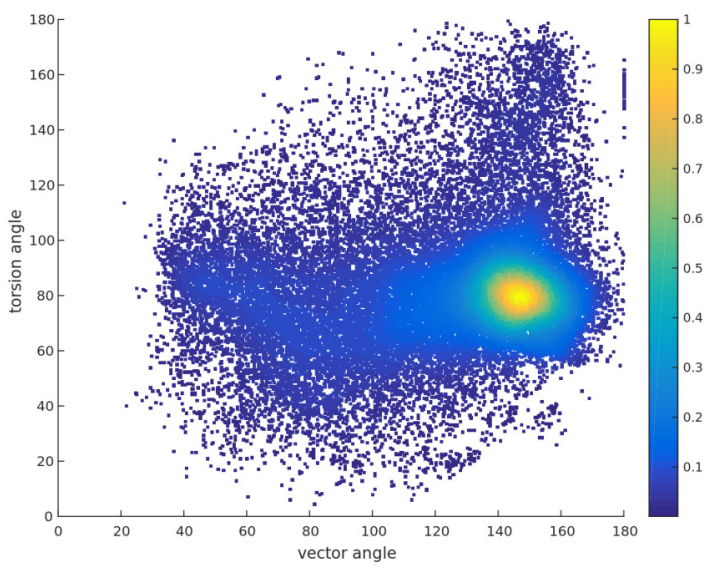

Fig. 6. Two scatterplots depicting the values of vector and torsion angles found in the representations of the Head and Hand joints. Points tending to the warmer colors correspond to pairs of angles that were found more frequently. The analysis reflects that the human hand is much more flexible than the head. 
ticle, at a mini-symposium ${ }^{4}$ focusing, among the other topics, on dynDGP applications. The reported animations (the same examples discussed here are presented) show that fluid motions can be obtained by our revisited distance-based approach.

Finally, one may wonder whether we can still obtain such good results when the modifications on the bone lengths are more important (more than $30 \%$ modification of the original length). In this case, as expected, the results get worse and worse, in general. In order to better deal with these more important changes, we have implemented an intermediate skeleton approach (see Fig. 5). The idea of using intermediate skeletons to improve the results of motion retargeting was initially proposed in [14] in an inverse kinematics approach; we have simply re-implemented it in our contest. Instead of attempting to retarget a motion with large morphology changes, the idea is to perform intermediate retargetings in a sequence, in order to smoothly approach to the desired morphology. Every skeleton in the sequence has an intermediate morphology between the original $\chi$ and the target $\hat{\chi}$. In the experiment depicted in Fig. 5, we can see that the intermediate skeleton approach can actually improve the quality of our retargetings when the changes on the morphology are more important.

\section{AN ANALYSIS ON A DATABASE OF HUMAN MOTIONS}

In this section, we present a statistical analysis on the vector and torsion angles that we used in the new character representation presented in Section III, through the function $\rho^{\prime}$. To perform the analysis, we consider a similar idea proposed in [20] for analyzing the two main torsion angles involved in the conformation of an amino acid in a protein backbone. In the studied molecules, in fact, the vector angles are considered as constants (this is a hypothesis that does not apply to animated characters, see discussion in Section III). Here, instead of studying subsets of atoms (the ones belong to an entire amino acid), we focus our attention on single joints, where we compare (we plot) the possible vector angles, related to the joint, against the corresponding torsion angles.

To perform our analysis, we consider the same database of human motions from which we selected the motions for our experiments in Section IV-A. This database consists of many motions with varying lengths (in terms of frames). Instead of preforming the analysis on the full motions of the database, we randomly selected $1 \%$ of the frames composing all the motions present in the database. We repeated this analysis several times to verify that the results were representative of the complete database. A useful observation from the analysis is that some joints have rather limited movement possibilities while others are much more flexible. Moreover, the analysis shows that simple constraints can potentially be introduced on the vector and torsion angles to constrain the characters to take

${ }^{4}$ Mini-symposium on Sensor Network Localization and Dynamical Distance Geometry, scheduled as part of Thematic Program on Geometric Constraint Systems, Framework Rigidity, and Distance Geometry, Fields Institute, Toronto, Canada, May 18-27, 2021.
Leg joint (left or right)

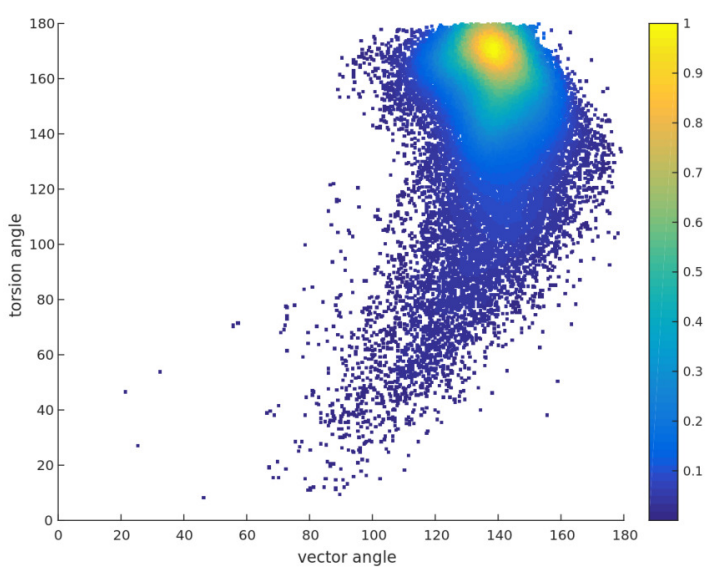

Foot joint (left or right)

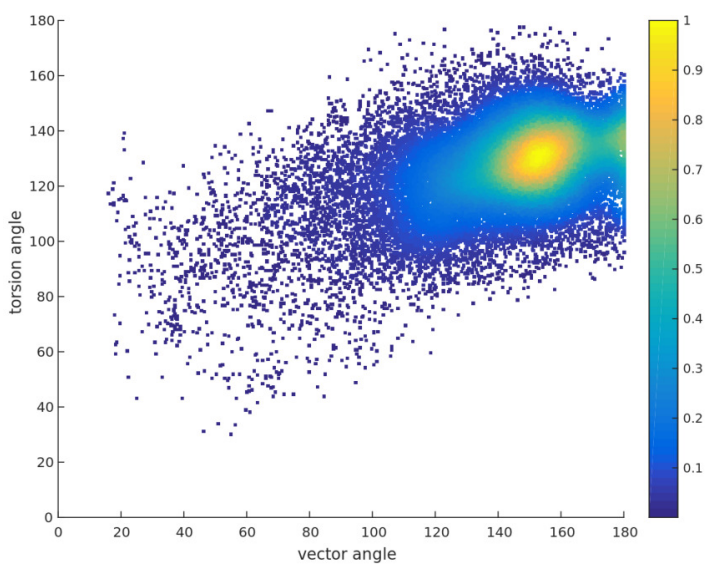

Fig. 7. Two other scatterplots related to the Leg and Foot joints.

only natural postures. This can potentially help our distancebased approach avoiding unnatural postures, especially when the changes in the morphology are more important.

In Fig. 6, we compare the plot corresponding to the angles obtained while analyzing the Head joint to the plot obtained for the Hand joint. As one may have expected, the figure shows that the human hand has a quite large flexibility (almost the entire space is feasible), but we can identify a sub-region (in warmer colors in the figure) where the pairs of angles are more frequent. The joint representing the head of the character has instead much more limited movement possibilities. In the hypothesis the considered motion database actually covers all human suitable movements, we can therefore impose rather tight constraints to joints (such as the Head joint) in the $\rho^{\prime}$ representation, in order to ensure that the corresponding movements always look natural.

Fig. 7 shows two other plots, one obtained by analyzing the first joint representing the character legs (the parents are the hips), and another obtained by analyzing the character feet. Both leg and feet joints, on the left or right side of 
the character, define similar plots. Again, these plots present several combinations of angles (the white regions in the plot) that are never reached.

\section{CONCLUSIONS}

We have revisited a distance-based approach to human motion adaptation, and presented a statistical analysis to establish the joint movements that allow the character to perform natural motions. To this purpose, we have discussed some alternative representations for the character motions. While we used the most popular representation (based on Euler angles) in our experiments on motion adaptation, we have found out that this may not be the most efficient one, and proposed an alternative representation, which we then used in our statistical analysis.

Future works will include a wider testing of this novel approach, with more human motions, and with several simultaneous changes (even asymmetric) performed on the morphology of the target character. Moreover, we will perform a deeper study on the several representations of the motions presented in this article, and we will compare each against the other. Currently, our results seem to suggest that the most efficient representation is the one introduced in Section III, and the result of our statistical analysis seem to validate this fact. However, this efficiency is currently supported, from a theoretical point of view, only by a simple study on its degrees of freedom, and therefore additional investigations in this direction need to be performed.

\section{Acknowledgments}

We are grateful to the anonymous reviewers for their comments on the initial version of this manuscript. This work is partially supported by the international project MULTIBIOSTRUCT funded by the ANR French funding agency (ANR-19-CE45-0019).

\section{REFERENCES}

[1] A. Alfakih, Universal Rigidity of Bar Frameworks in General Position: a Euclidean Distance Matrix Approach. In: [18], Springer, 3-22, 2013.

[2] H. Berman, J. Westbrook, Z. Feng, G. Gilliland, T. Bhat, H. Weissig, I. Shindyalov, P. Bourne, The Protein Data Bank, Nucleic Acids Research 28, 235-242, 2000.

[3] A. Bernardin, L. Hoyet, A. Mucherino, D.S. Gonçalves, F. Multon, Normalized Euclidean Distance Matrices for Human Motion Retargeting, ACM Conference Proceedings, Motion in Games 2017 (MIG17), Barcelona, Spain, November 2017.

[4] J. Diebel, Representing Attitude: Euler angles, Unit Quaternions, and Rotation Vectors, Matrix 58(15-16), 1-35, 2006.
[5] R. Featherstone, Rigid Body Dynamics Algorithms, Springer, 279 pages, 2008.

[6] M. Gleicher, Retargetting Motion to New Characters. ACM Proceedings of the $25^{t h}$ annual conference on Computer Graphics and Interactive Techniques, 33-42, 1998.

[7] S. Guo, R. Southern, J. Chang, D. Greer, J.J. Zhang, Adaptive Motion Synthesis for Virtual Characters: a Survey, The Visual Computer 31(5), 497-512. 2015.

[8] E.S.L Ho, T. Komura, C-L. Tai, Spatial Relationship Preserving Character Motion Adaptation, Proceedings of the $37^{\text {th }}$ International Conference and Exhibition on Computer Graphics and Interactive Techniques, ACM Transactions on Graphics 29(4), 8 pages, 2010.

[9] G. Laman, On Graphs and Rigidity of Plane Skeletal Structures, Journal of Engineering Mathematics 4(4), 331-340, 1970.

[10] L. Liberti, C. Lavor, N. Maculan, A. Mucherino, Euclidean Distance Geometry and Applications, SIAM Review 56(1), 3-69, 2014.

[11] T.E. Malliavin, A. Mucherino, M. Nilges, Distance Geometry in Structural Biology: New Perspectives. In: [18], Springer, 329-350, 2013.

[12] W. Maurel, D. Thalmann, Human Shoulder Modeling Including ScapuloThoracic Constraint and Joint Sinus Cones, Computers \& Graphics 24, 203-218, 2000.

[13] M. Meredith, S. Maddock, Motion Capture File Formats Explained, Technical Report 211, Department of Computer Science, University of Sheffield, 36 pages, 2001.

[14] J.-S. Monzani, P. Baerlocher, R. Boulic, D. Thalmann, Using an Intermediate Skeleton and Inverse Kinematics for Motion Retargeting, Computer Graphics Forum 19(3), 11-19, 2000.

[15] A. Mucherino, Introducing the Interaction Distance in the context of Distance Geometry for Human Motions, Chebyshevskii sbornik 20(2), 263-273, 2019.

[16] A. Mucherino, D.S. Gonçalves, An Approach to Dynamical Distance Geometry, Lecture Notes in Computer Science 10589, F. Nielsen, F. Barbaresco (Eds.), Proceedings of Geometric Science of Information (GSI17), Paris, France, 821-829, 2017.

[17] A. Mucherino, D.S. Gonçalves, A. Bernardin, L. Hoyet, F. Multon, A Distance-Based Approach for Human Posture Simulations, IEEE Conference Proceedings, Federated Conference on Computer Science and Information Systems (FedCSIS17), Workshop on Computational Optimization (WCO17), Prague, Czech Republic, 441-444, 2017.

[18] A. Mucherino, C. Lavor, L. Liberti, N. Maculan (Eds.), Distance Geometry: Theory, Methods and Applications, 410 pages, Springer, 2013.

[19] A. Mucherino, J. Omer, L. Hoyet, P. Robuffo Giordano, F. Multon, An Application-based Characterization of Dynamical Distance Geometry Problems, Optimization Letters 14(2), 493-507, 2020.

[20] G.N. Ramachandran, C. Ramakrishnan, V. Sasisekharan, Stereochemistry of Polypeptide Chain Configurations, Journal of Molecular Biology 7, 95-104, 1963.

[21] J. Saxe, Embeddability of Weighted Graphs in $k$-Space is Strongly NPhard, Proceedings of $17^{\text {th }}$ Allerton Conference in Communications, Control and Computing, 480-489, 1979.

[22] G.G. Slabaugh, Computing Euler Angles from a Rotation Matrix, Technical Report, City University London, 8 pages, 1999.

[23] P. Tabaghi, I. Dokmanić, M. Vetterli, Kinetic Euclidean Distance Ma trices, IEEE Transactions on Signal Processing 68, 452-465, 2020. 